\title{
DNA Endoreplication in the Brain Neurons during Body Growth of an Adult Slug
}

\author{
Miki Yamagishi, Etsuro Ito, and Ryota Matsuo \\ Kagawa School of Pharmaceutical Sciences, Tokushima Bunri University, Sanuki, Kagawa 769-2193, Japan
}

Endoreplication is DNA synthesis without cell division. Giant neurons observed in the brains of mollusks are thought to be generated as a result of DNA endoreplication. It has been hypothesized that neuronal size becomes larger in parallel with an increase in body size and that DNA endoreplication is involved in this process to meet the increasing demand for macromolecules in neurons. There is, however, no experimental evidence for this hypothesis to date. In the present study, we investigated the following quantitatively: (1) the size of the brain and each ganglion, (2) the size of identified neurons, (3) the total number of neurons undergoing DNA endoreplication, (4) the total number of the neurons containing a cardioexcitatory peptide, and (5) the gene expression level per neuron, using terrestrial slugs whose body growth was regulated through the amount of food supplied in the laboratory. The body growth was accompanied by increases in the sizes of both neurons and ganglia and triggered more frequent DNA endoreplication events in each ganglion of the growth-promoted slugs, without increasing the total number of neurons. Increase in the neuronal size also involved the increase in the amount of transcripts expressed in a single neuron. This is the first quantitative evidence showing that the DNA endoreplication, neuronal size, and gene expression are increased concomitantly with body growth in adult mollusks.

\section{Introduction}

Most of the somatic cells of animals have primarily diploid genomes in their nuclei. This is especially the case for the neurons in the brains of vertebrates, although there are a small number of exceptions in the neuroendocrine systems of the teleost (Mola et al., 2001; Cuoghi and Mola, 2007). Conversely, it has been recognized for some time that the brain of the gastropod mollusk contains many giant neurons whose nuclei are greatly enlarged. Lasek and Dower (1971) quantified the amount of DNA in the nucleus of a single giant neuron of Aplysia and found that it is equivalent to more than several hundreds of thousand times as much DNA as the haploid genome (Lasek and Dower, 1971). Similarly, a large amount of DNA is also contained in the nuclei of the giant neurons in Helix (Kuhlmann, 1969), Lymnaea (Boer et al., 1977), and Achatina (Chase and Tolloczko, 1987). Such a genome is postulated to be produced as a result of repeated endoreplication, although it is still controversial whether the endoreplication is whole DNA replication (polyploidy) or localized DNA amplification, restricted to specific genomic regions (polyteny) (Mandrioli et al., 2010).

As an explanation for this phenomenon, Gillette (1991) proposed that DNA endoreplication results in the giantism of

\footnotetext{
Received Jan. 11, 2011; revised Feb. 3, 2011; accepted Feb. 7, 2011.

Author contributions: R.M. designed research; M.Y. and R.M. performed research; M.Y., E.I., and R.M. analyzed data; E.I. and R.M. wrote the paper.

This study was supported by Japan Society for the Promotion of Science KAKENHI Grants-in-Aid 22570077 (R.M.) and 19370030 and 21657022 (E.I.), a Narishige Zoological Science Award (R.M.), and a Grant for Joint Research Programs of Tokushima Bunri University (R.M.). We thank Fumihiro Morishita for providing the antibody against NdWFamide and Hisayo Sadamoto for technical advice on quantitative real-time PCR analysis.

Correspondence should be addressed to Dr. Ryota Matsuo, Laboratory of Functional Biology, Kagawa School of Pharmaceutical Sciences, Tokushima Bunri University, 1314-1 Shido, Sanuki, Kagawa 769-2193, Japan. E-mail: matsuor@kph.bunri-u.ac.jp.

DOI:10.1523/JNEUROSCI.0179-11.2011

Copyright $\odot 2011$ the authors $\quad 0270-6474 / 11 / 315596-09 \$ 15.00 / 0$
}

neurons, especially in motor neurons, to meet the increasing demands for innervating larger areas of the growing body and therefore to produce a larger amount of macromolecules for the functioning of these motor neurons. This plausible explanation has, however, not been tested experimentally to date, nor has even evidence for DNA synthesis in the neurons of adult mollusks been reported yet. We thus investigated this hypothesis quantitatively using the terrestrial slug Limax valentianus in the present study. The slugs were maintained in our laboratory under regulated conditions from birth to death. We prepared two groups of slugs, maintained under two extreme conditions to differentiate their body sizes: (1) access to food ad libitum (growth promotion) or (2) completely starved for $44 \mathrm{~d}$ (growth suppression). We then quantitatively compared the following: (1) the size of the brain and each ganglion, (2) the size of the identifiable neurons, (3) the total number of a specific type of neurons, (4) the frequency of DNA synthesis in neurons, and (5) the expression level of a specific transcript per neuron. Our results demonstrated that the body growth is accompanied by DNA synthesis and each neuron is enlarged without increasing the number of neurons. These neurocytological changes result in the enlargement of the whole brain and involve increased gene products in each neuron. This is the first experimental evidence supporting the hypothesis that body growth affects the neuronal size through DNA endoreplication.

\section{Materials and Methods}

Animals. The terrestrial slugs Limax valentianus were maintained in our laboratory at $19^{\circ} \mathrm{C}$ for at least 11 generations as a closed colony. They were fed on a diet of humidified powder mixture (for its composition, see Fukunaga et al., 2006).

Dietary regulation of slugs. The slugs (10-11 weeks after hatching) were housed individually in separate plastic containers $(83 \times 62 \times 20 \mathrm{~mm})$ in 
which a moistened filter paper (Advantec) was laid. For the growthpromoted or the normally grown (control) groups of slugs, $\sim 0.4$ or $0.02 \mathrm{~g}$, respectively, of the humidified powder mixture food was supplied every other day for $44 \mathrm{~d}$. For the growth-suppressed group of slugs, no food was provided during this period. It has been reported that the slugs are almost sexually mature at the age of 10-11 weeks after hatching, although the speed of maturation depends on the temperature and the photoperiod during rearing (Udaka and Numada, 2008).

Toluidine blue staining and volume measurement. Forty-four days after the beginning of the dietary regulation, the slugs were deeply anesthetized by an injection of $\sim 500 \mu \mathrm{l}$ of ice-cold $\mathrm{Mg}^{2+}$ buffer (in mM: 57.6 $\mathrm{MgCl}_{2}, 5$ glucose, and 5.0 HEPES, $\mathrm{pH}$ 7.0) into the body cavity, and the brain was dissected out. The dissected brain was frozen in Tissue-Tek optimal cutting temperature compound (Sakura) using liquid nitrogen. Cryostat sections ( $14 \mu \mathrm{m}$ thick) were cut coronally all through the brain (except the buccal ganglion) and mounted onto glass slides coated with Vectabond (Vector Laboratories). The sections were fixed in 10\% formaldehyde neutralized buffer (Nakarai-Tesque) for $30 \mathrm{~min}$ at room temperature and stained with $0.02 \%(\mathrm{w} / \mathrm{v})$ toluidine blue solution, followed by several washes in water. The sections were coverslipped with Permount (Biomeda). The images of the stained sections were obtained with a light microscope BX-51 (Olympus) equipped with a CCD camera DP70 (Olympus) and an objective ( $4 \times, 0.16$ numerical aperture). The areas (square micrometers) of the cell bodies and neuropiles were measured using the software MetaMorph version 6.1 (Molecular Devices). Because the neuropile area of the procerebrum (PC) is continuous with that of the medial part of the cerebral ganglia, the boundary was set in each coronal section based on the slight difference in the staining intensity between the two neuropile areas. The measured areas were multiplied by the thickness of the sections $(14 \mu \mathrm{m})$ and then summed to calculate the volume of each ganglion. For the pedal, pleural, and cerebral (without PC) ganglia and the PC, the volumes of the bilateral ganglia were integrated. The parietal and visceral ganglia were considered to be one structure because these ganglia were structurally not separable in several sections.

Estimation of the relative number of neurons in the pleural ganglion. The left pleural ganglion in the photographs of the toluidine blue-stained sections was analyzed for the growth-promoted and the growthsuppressed slugs. Those sections were chosen that contained neurons with relatively uniform diameters and that displayed the equivalent planes of the brain in the growth-promoted $(n=4)$ and the growthsuppressed $(n=4)$ slugs. The area density $\left(d_{s}\right)$ of the number of neurons was calculated based on the images of these sections, and the 1.5 th power of the ratio of $d_{s}$ between the growth-suppressed and the growthpromoted $\left[\left(d_{s \text {-suppressed }} / d_{s \text {-promoted }}\right)^{3 / 2}\right]$ slugs was calculated to estimate the ratio of the volume density of the number of neurons $\left(d_{v \text {-suppressed }}\right.$ / $\left.d_{v \text {-promoted }}\right)$. This ratio was multiplied by the ratio of the volume of the cell body areas in the pleural ganglia between the growth-suppressed and the growth-promoted slugs $\left(v_{\text {suppressed }} / v_{\text {promoted }}\right.$; see above) to see whether there was a difference in the total number of neurons included in the left pleural ganglia between the growth-promoted and the growthsuppressed slugs.

Extraction of genomic DNA. Genomic DNA was extracted from each brain using proteinase $\mathrm{K}$ and phenol/chloroform/isoamylalcohol as described by Sambrook and Russell (2001). The extracted DNA was treated with RNase A, which was later removed by phenol/chloroform/isoamylalcohol. We used Ethatinmate (Wako Pure Chemicals) to improve the efficiency of DNA recovery during the ethanol precipitation. DNA was dissolved in 5-10 $\mu$ l of TE (10 mm Tris and 1 mm EDTA, pH 7.5) and was quantified based on the light absorbance (wave length, $260 \mathrm{~nm}$ ).

5-Bromo-2'-deoxy-uridine labeling and immunostaining. 5-Bromo-2'deoxy-uridine (BrdU) was injected $14 \mathrm{~d}$ after the beginning of the dietary regulation. BrdU was dissolved (0.5 mM) in Limax physiological buffer solution (in mu: $70 \mathrm{NaCl}, 2.0 \mathrm{KCl}, 4.7 \mathrm{MgCl}_{2}, 4.9 \mathrm{CaCl}_{2}, 5.0$ glucose, and 5.0 HEPES, pH 7.0) and was injected into the body cavity $(0.5 \mathrm{ml} /$ gross body weight) of the adult slugs twice, with an interval of $1 \mathrm{~h}$. After $30 \mathrm{~d}$, these slugs were deeply anesthetized by an injection of $\sim 500 \mu \mathrm{l}$ of icecold $\mathrm{Mg}^{2+}$ buffer into the body cavity, and the brain was dissected out. The dissected brain was frozen as above, and cryostat sections $(20 \mu \mathrm{m}$ thick) were cut coronally all through the brain and mounted onto glass slides coated with Vectabond. We used 5-Bromo-2'-deoxy-uridine Labeling and Detection Kit I (Roche Diagnostics). Immunostaining was performed according to the instructions of the manufacturer with slight modifications. Briefly, the sections were fixed with ethanol fixative (50 mu glycine and $70 \%$ ethanol solution, $\mathrm{pH} 2.0$ ) for $20 \mathrm{~min}$ at $-20^{\circ} \mathrm{C}$. After washing by several changes of PBS for rehydration, the sections were incubated for $30 \mathrm{~min}$ at $37^{\circ} \mathrm{C}$ in diluted (1:10) mouse monoclonal antiBrdU antibody containing deoxyribonuclease (DNase) in incubation buffer (in mM: 66 Tris, pH 8.4, $0.66 \mathrm{MgCl}_{2}$, and 1.0 2-mercaptoethanol]. After washing in PBS, the sections were incubated with Alexa Fluor 488labeled secondary anti-mouse IgG antibody (1:500 in PBS; Invitrogen) for $1 \mathrm{~h}$ at room temperature. After several washes in PBS, the cover glasses were mounted with Vectashield with $4^{\prime}, 6^{\prime}$-diamidino-2-phenylindole (DAPI) (Vector Laboratories). The fluorescence images were obtained with a fluorescence microscope (BX-51; Olympus) equipped with a CCD camera (DP70; Olympus). The number of BrdU-positive nuclei were counted and summed all through the sections of each ganglion. The very-small-diameter BrdU-positive spots (less than $\sim 10 \mu \mathrm{m}$ ), probably reflecting non-neuronal cells (epithelial cells of the brain sheath or glial cells) or debris, were excluded from counting.

Immunohistochemistry of NdWFamide. Rabbit polyclonal antiNdWFamide antibody (Morishita et al., 2001, 2003a) was provided by courtesy of Dr. Fumihiro Morishita (Hiroshima University, Hiroshima, Japan). The isolated brain of a slug was fixed in Bouin fixative $(2.9 \%$ picric acid, $8.8 \%$ formaldehyde, and $4.8 \%$ acetic acid) for $3-4 \mathrm{~h}$ at room temperature. After the treatment in $70 \%$ ethanol at $4^{\circ} \mathrm{C}$ overnight, the fixed brain was cryoprotected in $20 \%$ sucrose in PBS at $4^{\circ} \mathrm{C}$ overnight. The brain was frozen in Tissue-Tek optimal cutting temperature compound using liquid nitrogen. Cryostat sections ( $14 \mu \mathrm{m}$ thick) were cut coronally and mounted onto glass slides coated with Vectabond. After the postfixation in $5 \%$ formaldehyde neutralized buffer (the above $10 \%$ formaldehyde neutralized buffer was diluted to one-half by PBS) for 30 min at room temperature, the sections were treated with $0.1 \%$ Triton $\mathrm{X}-100$ in PBS (PBST) for 10 min. After a brief wash in PBS, the sections were incubated in blocking buffer $(2.5 \%$ goat serum and $2.5 \%$ bovine serum albumin in PBST) at $4^{\circ} \mathrm{C}$ overnight. The sections were then incubated with polyclonal anti-NdWFamide antibody (1:400 in blocking buffer) for $1 \mathrm{~h}$ at room temperature. After three washes in PBS, the sections were incubated with a secondary antibody against rabbit IgG labeled with Alexa Fluor 488 (1:500 in blocking buffer; Invitrogen) for $1 \mathrm{~h}$ at room temperature. After two washes in PBS, the sections were incubated in 0.1 $\mu \mathrm{g} / \mathrm{ml}$ DAPI in PBS for $10 \mathrm{~min}$ and then washed once in PBS. The sections were coverslipped with Vectashield with DAPI (Vector Laboratories). The fluorescence images were obtained with a fluorescence microscope (BX-51) equipped with a CCD camera (DP70). The specificity of the primary antibody was confirmed by preincubation of antiNdWFamide antibody (1:400 in blocking buffer) with $0.22 \mathrm{~mm}$ NdWFamide peptide at $4^{\circ} \mathrm{C}$ overnight.

In situ hybridization of achatin-I mRNA. Full-length achatin-I cDNA (GenBank accession number AB598836) cloned into pBluescript II (Stratagene) was digested with EcoRI or XhoI to prepare the linearized template DNA for the antisense or the sense riboprobe, respectively. The template DNA was transcribed in the presence of digoxigenin (DIG) RNA labeling mix (Roche Diagnostics) using T7 or T3 RNA polymerase to synthesize the antisense or the sense riboprobe, respectively. The working concentrations of cRNA probes were determined by titration to ensure the equivalence of the DIG incorporation into the antisense and sense probes. Hybridization and wash were performed as described previously (Matsuo et al., 2009). The sections (50 $\mu$ m thickness) were imaged using a confocal microscope LMS510 (Carl Zeiss) with a $40 \times$ oil-immersion objective and were scanned differentially in depth with each image separated by $1 \mu \mathrm{m}$ from one end to the other. For the signal detection by color development, the thickness of sections was $14 \mu \mathrm{m}$, and 5-bromo-4-indolyl phosphate $p$-toluidine salt and 4-nitro blue tetrazolium chloride were used as the substrates for the alkaline phosphatase conjugated with anti-DIG antibody, as described previously (Matsuo et al., 2001).

Double staining of BrdU and neuropeptide mRNAs. The partial cDNA (145-543 bp) fragment of achatin-I was cloned into a pCRII TA cloning vector (Invitrogen), and the antisense and the sense riboprobes were 
prepared as described above. For the lightyellow cell (LYC) prepropeptide gene, the fulllength cDNA (GenBank accession number AB598837) cloned in pBluescript II was used as a template, and the antisense and the sense riboprobes were also prepared as described above. Fluorescent double staining of BrdU and mRNAs of these neuropeptides was performed as described previously (Matsuo et al., 2010). The coronal sections (14 $\mu \mathrm{m}$ thickness) were imaged using a BX-51 fluorescence microscope with an attached DP70 CCD camera or a LMS510 confocal microscope with a $40 \times$ oil-immersion objective. The images of in situ hybridization and BrdU immunostaining were superimposed using Photoshop CS2 (Adobe Systems) software.

Reverse transcription-PCR of achatin-I. Expression analysis in brain regions by reverse transcription (RT)-PCR was performed as described previously for achatin-I and $18 \mathrm{~S}$ rRNA (Fukunaga et al., 2006). The nucleotide sequences of PCR primers for achatin-I were as follows: upstream primer, $5^{\prime}$ GGCAGTGGCATTGAACCTC-3'; downstream primer, 5'-GCTATCATCCCATCACATCG-3' .

The PCR products were electrophoresed in a $1 \%(\mathrm{w} / \mathrm{v})$ agarose gel and visualized using ethidium bromide under a UV illuminator.

Quantitative real-time $R T$-PCR. The total RNA was extracted using an acid-guanidinium thiocyanate-phenol-chloroform method (Chomczynski and Sacchi, 1987) from each brain of the slugs that were killed $44 \mathrm{~d}$ after the dietary regulation (see above). After DNase I treatment, $0.4 \mu \mathrm{g}$ of RNAs were reverse transcribed using Moloney murine leukemia virus reverse transcriptase (Invitrogen) and oligo-dT primer in a $20 \mu \mathrm{l}$ of reaction mixture. An aliquot $(1.0 \mu \mathrm{l})$ of the cDNA was used as a template for PCR amplification. The PCR amplification was performed using Syber Premix Ex TaqII reagent (Takara) in a 7900HT fast real-time PCR system (Applied Biosystems) according to the instructions of the manufacturer. To calibrate the copy numbers of achatin-I cDNA, serial diluents $\left(10^{7}, 10^{6}, 10^{5}\right.$, $10^{4}, 10^{3}$, and 0 copies) of full-length cDNA (in pBluescript II) were prepared, and they were also amplified in parallel. All cDNA samples were amplified in duplicate, and the average values were adopted. The nucleotide sequences of the PCR primers were as follows: upstream primer, 5' -ATCAAGGAACGGGACACAGTTC-3'; downstream primer, 5'-GAAGCCAATTTGGGAATCTGATG-3'.

The calculated copy numbers of achatin-I cDNAs in each PCR tube were extrapolated to the total copy numbers in each brain based on the amount of RNA used for reverse transcription.

Statistical analysis. The data for two groups were statistically analyzed by Student's or Welch's two-tailed $t$ test with a significance level of $p<0.05$. The data for multiple groups were statistically analyzed by a one-way ANOVA or a Kruskal-Wallis test. The post hoc test we used was the Scheffe's test with a significance level of $p<0.05$. The data are expressed as the mean \pm SE throughout.

\section{Results}

\section{Brain size is increased during the gain of body weight}

If the size of each neuron is increased as the animal grows, the total size of the brain should also be increased. To investigate this possibility, we prepared the growth-promoted and the growth-suppressed slugs by dietary regulation. As a result of $44 \mathrm{~d}$ of dietary regulation, the body weight of the ad libitum food-access (growthpromoted) slugs became $\sim 10$ times greater than before the dietary regulation, whereas that of the starved (growth-suppressed) slugs was unchanged (Fig. 1A,B). The normally fed (control) slugs grew only slightly (Fig. $1 B$ ). At $44 \mathrm{~d}$ after the dietary regulation, we dis-
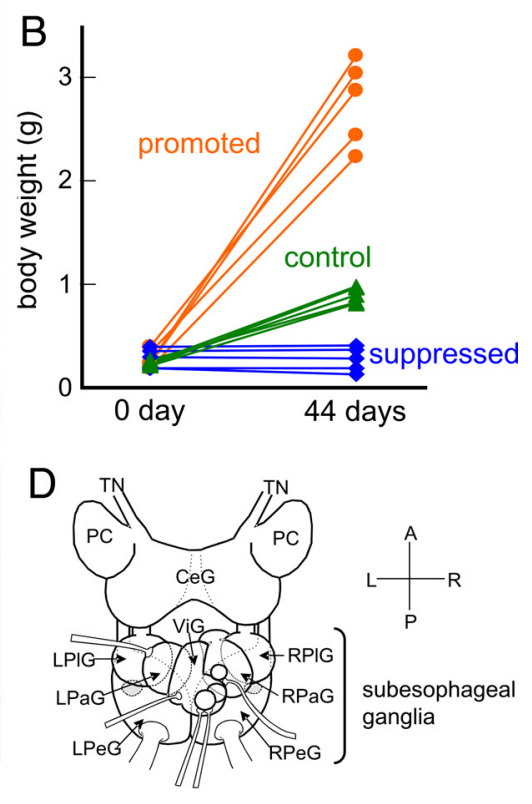

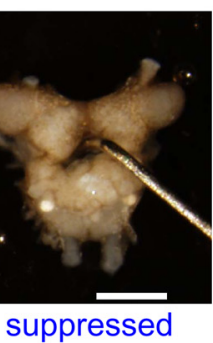

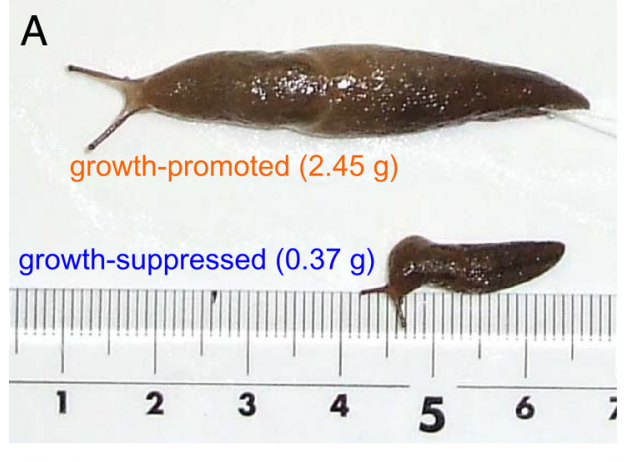



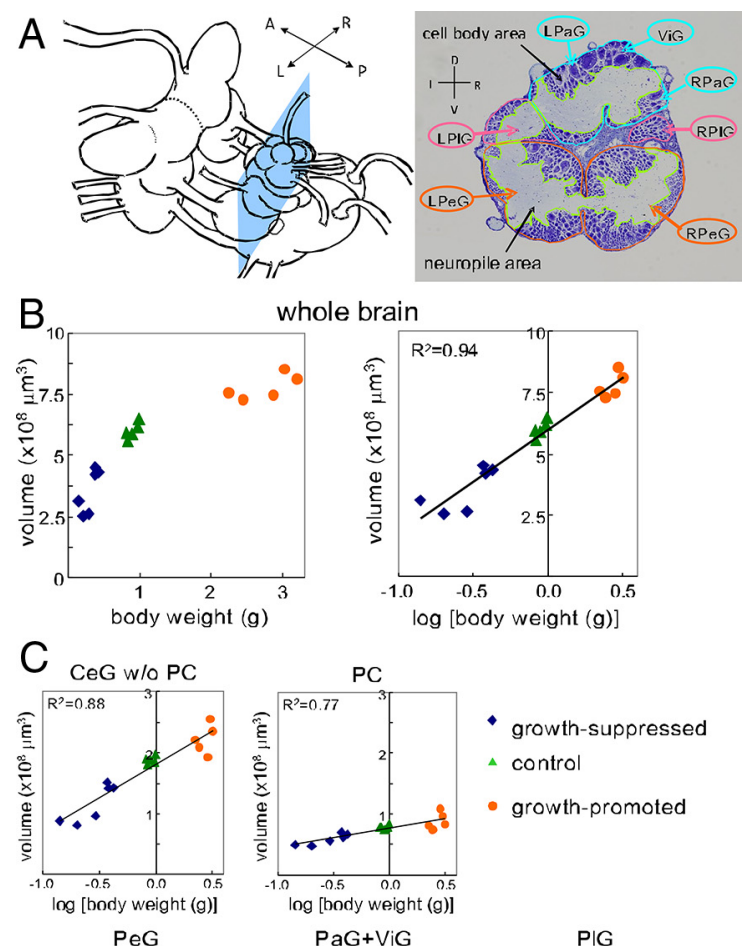

- growth-suppressed

A control

- growth-promoted
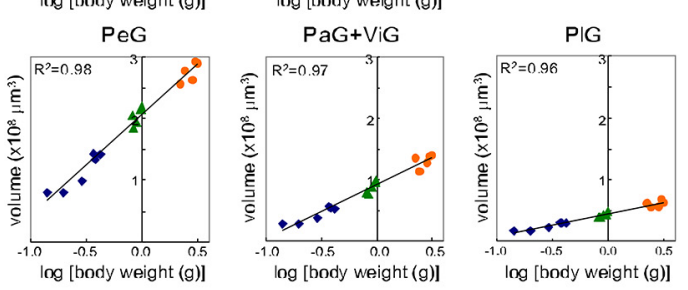

D $\square$ growth-suppressed $\square$ control $\square$ growth-promoted
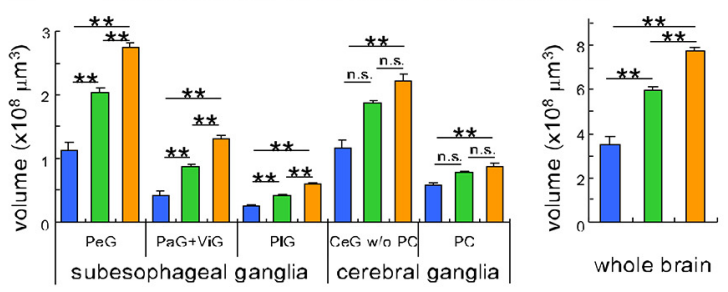

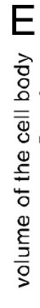
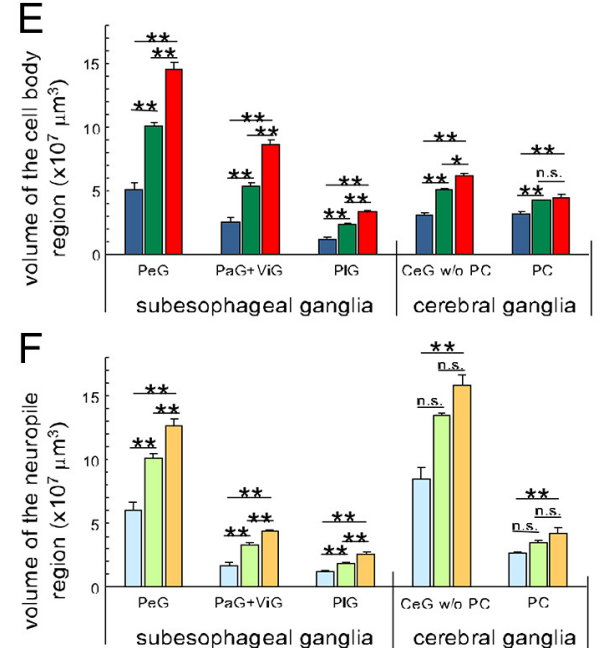

Figure 2. Correlation between the body weight and brain size. $\boldsymbol{A}$, The area measurement in a toluidine-blue-stained section. The right corresponds to the coronal section as shown in the left diagram. The area was measured for the cell body and neuropile regions for each ganglion. D, Dorsal; V, ventral; R, right; L, left. $\boldsymbol{B}$, Positive correlation between the body weight and the brain volume. There was a linear relationship between the logarithm of the body weight and the ral, 2.78 vs 2.14; cerebral without PC, 2.01 vs 1.86 ) (Fig. 2E), whereas the increase was rather ascribable to that of the neuropile regions in the PC (cell body, 1.41-fold vs neuropile, 1.61-fold) (Fig. 2F).

To investigate whether the volume increase in the cell body region was caused by an increase of each neuron, we examined the sizes of two types of identifiable giant neurons (Fig. $3 G$ ), a pair of visceral giant neurons (VGCs) and a pair of metacerebral giant neurons (MGCs). The VGCs contain LdWFamide (Fig. 3A-F), which has a potent cardioexcitatory activity in mollusks (Morishita et al., 1997, 2003a,b), and these neurons are the putative homologs of those identified in the dorsal surface of the visceral ganglion of another pulmonate, Euhadra congenita (Morishita et al., 2003b). The MGCs are known to be serotonergic neurons involved in the feeding rhythm generation in gastropods (Weiss and Kupfermann, 1976; Yeoman et al., 1996; Shirahata et al., 2004). We chose these pairs of neurons because these were unambiguously identifiable in every slug. Based on the area of these neurons in the toluidine-blue-stained sections, the volumes of the cell bodies of these neurons was calculated; they proved to be larger in the growth-promoted slugs and smaller in the growthsuppressed slugs, showing a tendency similar to the case of the total volume of the ganglia (Fig. $3 H, I$ ).

To further examine whether or not the increase in the ganglion volume was caused by the increase in the number of neurons as well as in their sizes, we estimated the relative value of the total number of neurons within the left pleural ganglion between the growth-suppressed and the growth-promoted slugs. We chose this ganglion because neuronal size is relatively homogenous compared with the other ganglia. The average value of the relative area density of the number of neurons $\left(d_{s \text {-suppressed }} / d_{s \text {-promoted }}\right)$ was 1.84 , which is equivalent to the relative volume density $\left(d_{v \text {-suppressed }} / d_{v \text {-promoted }}\right)$ of $2.50\left(=1.84^{3 / 2}\right)$. Conversely, the relative volume of the cell body region in the pleural ganglion $\left(v_{\text {suppressed }} / v_{\text {promoted }}\right)$ was $0.36\left(1.21 \times 10^{7}\right.$ vs $\left.3.35 \times 10^{7} \mu^{3}\right)$ (Fig. $2 E$ ). Therefore, the relative value of the total number of neurons in the left pleural ganglion was estimated to be $d_{v \text {-suppressed }}$ $d_{v \text {-promoted }} \times v_{\text {suppressed }} / v_{\text {promoted }}=0.90$. This rough estimate indicated that there was not a large difference in the total number of neurons in the left pleural ganglion between the growthpromoted and the growth-suppressed slugs.

\section{DNA synthesis is enhanced during the gain of body weight}

If the giantism of neurons and brains is accompanied by DNA endoreplication in the nucleus of neurons, the total DNA content in the brain should be increased. We first tested this possibility by quantifying the amount of the genomic DNA in the whole brains of the growth-promoted and the growthsuppressed slugs. As shown in Figure $4 A$, the brains of the growth-promoted slugs contained significantly larger amounts of genomic DNA compared with that of the growth-suppressed slugs $(6.44 \pm 0.35$ vs $3.61 \pm 0.20 \mu \mathrm{g} /$ brain $)$, indicating that

brain volume (right). $C$, There were linear relationships between the logarithm of the body weight and the volume of each ganglion. $\boldsymbol{D}$, The mean volume of each ganglion in the growthsuppressed $(n=6)$, control $(n=5)$, and growth-promoted $(n=5)$ slugs. $\boldsymbol{E}$, The mean volume of the cell body region of each ganglion in the growth-suppressed, control, and growthpromoted slugs. $\boldsymbol{F}$, The mean volume of the neuropile region of each ganglion in the growthsuppressed, control, and growth-promoted slugs. ${ }^{* *} p<0.01$. PC, Procerebrum; $\mathrm{CeG}$, cerebral ganglion; ViG, visceral ganglion; PIG, pleural ganglion; $\mathrm{PaG}$, parietal ganglion; $\mathrm{PeG}$, pedal ganglion; $A$, anterior; $P$, posterior; $R$, right; L, left; $n$.S., no significant difference. 
DNA synthesis is enhanced in the brain during the growth promotion.

We next visualized DNA synthesis that occurred during the dietary regulation. BrdU was injected $14 \mathrm{~d}$ after the beginning of the dietary regulation, and the brain was dissected out after $30 \mathrm{~d}$ (Fig. 4B). Usually this protocol labels only those neurons that underwent DNA synthesis within a couple of days after the BrdU injection (Matsuo et al. 2010; Matsuo and Ito, 2011). The number of BrdU-positive neurons was significantly larger in the growth-promoted slugs than in the growth-suppressed slugs in all the ganglia examined, although the difference was less in the cerebral ganglia (Fig. 4C,D). We excluded the PC from the analysis because neurogenesis continuously occurs in the PC even in the adult (Zakharov et al., 1998; Watanabe et al., 2008), and it results in BrdU incorporation into many newborn neurons in the absence of DNA endoreplication (Matsuo and Ito, 2011). Most of the BrdU-positive signals were spatially sporadic and rarely existed in juxtaposed locations (Fig. 4C), indicating that these BrdU signals reflect DNA synthesis without cell division, that is, endoreplication.

\section{DNA synthesis occurs in some endocrine neurons}

To investigate in what type of neurons the DNA synthesis occurs, we performed double staining of incorporated BrdU in the nucleus and mRNA in the cytosol of neurons in the growth-promoted slugs. Achatin-I is another potent cardioexcitatory neuropeptide expressed in the subpopulation of neurons located in the ventroposterior part of the pedal ganglion (Satake et al., 1999) (Fig. 5A-C). LYC peptide is thought to be involved in the regulation of blood pressure and urine release in pulmonates and is specifically expressed in the right parietal ganglion (Boer and Montagne-Wajer, 1994) (Fig. 5D,E). We prepared cRNA probes for these neuropeptide genes and performed double staining of BrdU and these mRNAs in the coronal sections of the brain. Several neurons were doubly stained by the anti-BrdU antibody and the cRNA probes (Fig. $5 F-M$ ), indicating that some neuroendocrine cells exhibit DNA synthesis in the growthpromoted slugs during dietary regulation.

The number of achatin-I-positive neurons is invariant between the growth-promoted and the growth-suppressed slugs

To further examine the hypothesis that the increases in the DNA content of the brain (Fig. 4A) and in the size of the brain (Fig. 2) are not attributable to the cell division of neurons but are attributable, at least in part, to DNA endoreplication, we counted the total number of a single type of neurons within the brain. Because achatin-I mRNA is exclusively expressed in the ventroposterior right; L, left. Scale bar, $500 \mu \mathrm{m}$.
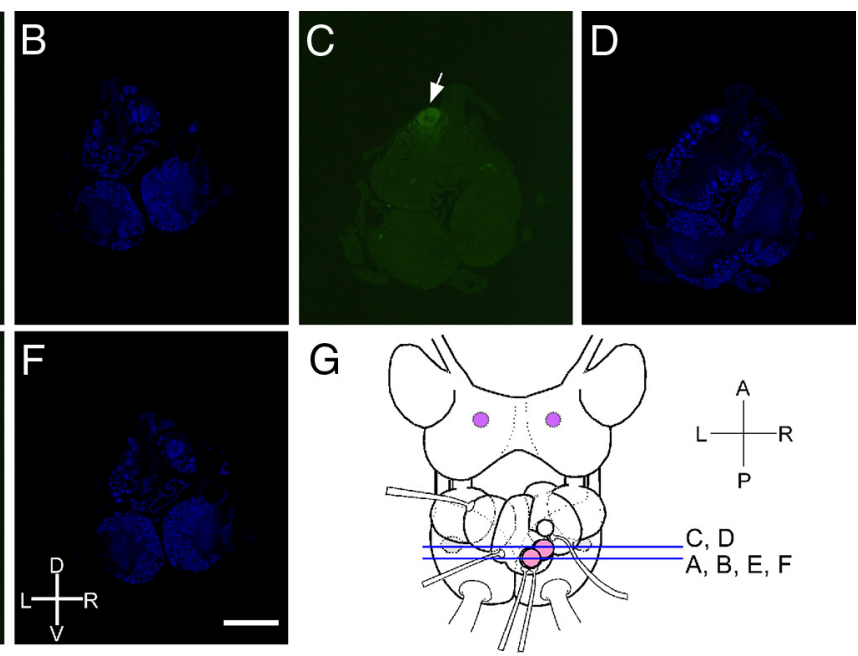

I

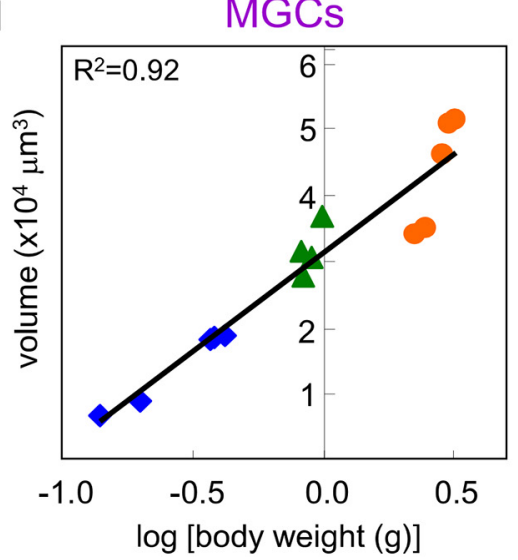

Figure 3. Correlation between the body weight and the size of the giant neurons. $A$, Immunostaining of one of the pair at the dorsal surface of the viscera ganglion, and MGCS are located near the ventral surface of the cerebral ganglia y the blue diamonds, green triangles, and orange circles, respectively. A, Anterior; P, posterior; D, dorsal; V, ventral; $R$,

part of the pedal ganglion (Fig. 5A-C) (Satake et al., 1999), we could count the total number of neurons that contain achatin-I mRNA in the growth-suppressed and growth-promoted slugs by scanning all the coronal sections including these neurons using confocal microscopy (Fig. 6A, B). There was no significant difference between the two groups (Fig. $6 C$ ), but we observed that the size of the achatin-I-positive neurons was larger in the growthpromoted slugs compared with the growth-suppressed slugs (Fig. $6 A, B)$. These results support the notion that the DNA synthesis does not reflect cell division but reflects DNA endoreplication within the nucleus of neurons.

Expression level of achatin-I mRNA per a single neuron is higher in the growth-promoted slugs than in the growth-suppressed slugs

Does DNA amplification result in increased gene products in each neuron? To address this issue, we measured the copy number of achatin-I transcripts using quantitative real-time RT-PCR. The absolute number of achatin-I cDNAs was determined for each brain from the growth-suppressed and growth-promoted 
A

B

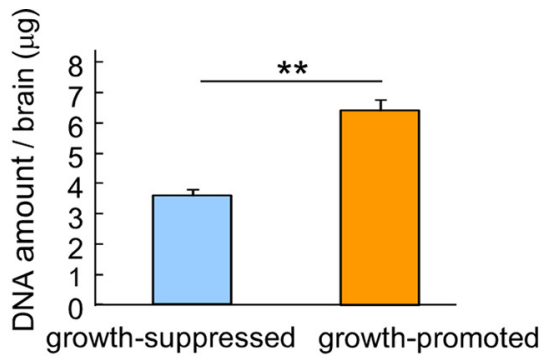

start of the

dietary regulation
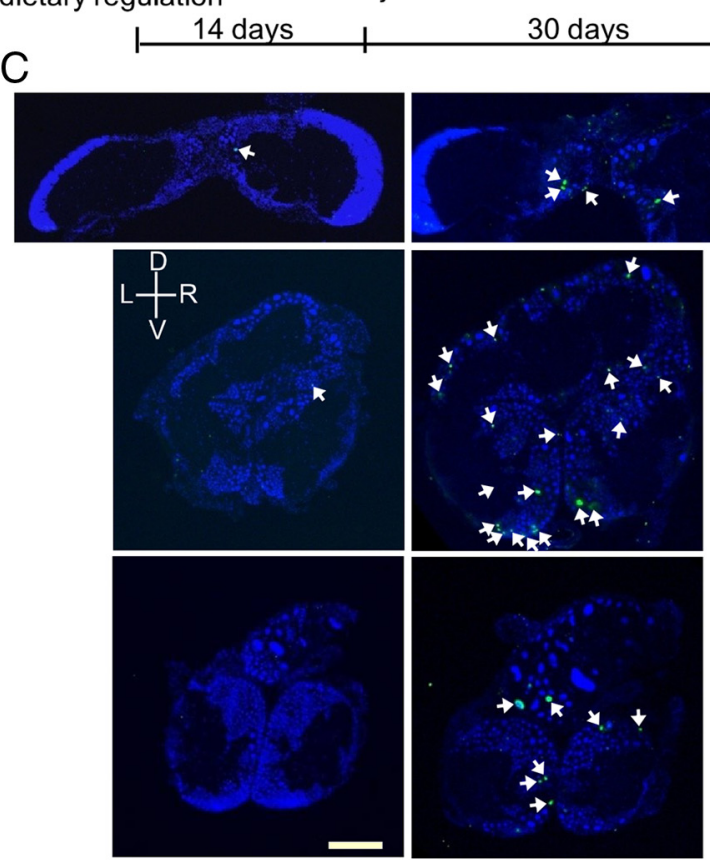

growth-suppressed

growth-promoted

D

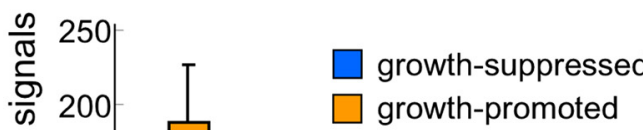

Figure 4. DNA endoreplication is more frequent in the brains of the growth-promoted slugs than in those of the growth-suppressed slugs. $A$, The total amount of genomic DNA was larger in the brains of the growth-promoted slugs than in those of the growthsuppressed slugs. $n=10$ each. ${ }^{* *} p<0.001$. B , Time schedule of the BrdU-labeling experiment. $C$, Examples of fluorescent microscopic images of the coronal sections stained by anti-BrdU antibody (green) and DAPI (blue). Top, Cerebral ganglia; middle, subesophageal ganglia showing the pedal, pleural, parietal, and visceral ganglia; bottom, subesophageal ganglia showing pedal and visceral ganglia. Arrows indicate BrdU-positive nuclei. Scale bar, $250 \mu \mathrm{m}$. D, Group data of the number of BrdU-positive neurons in each ganglion. Growth-suppressed slugs, $n=5$; growth-promoted slugs, $n=6$. ${ }^{*} p<0.05$, ${ }^{* *} p<0.005$. D, Dorsal; V, ventral; R, right; L, left; PeG, pedal ganglion; PaG, parietal ganglion; ViG, visceral ganglion; PIG, pleural ganglion; PC, Procerebrum; CeG, cerebral ganglion.
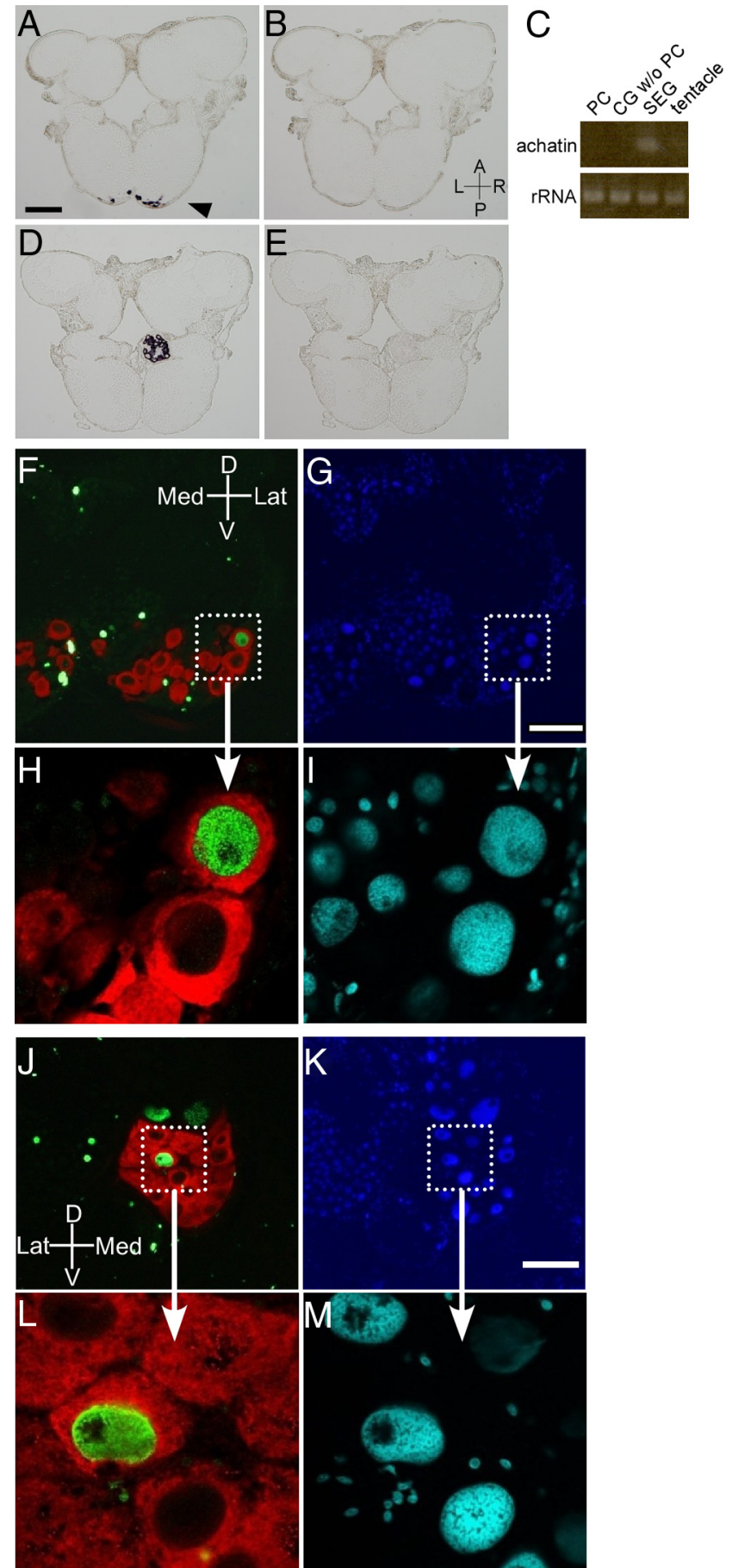

Figure 5. DNA synthesis in neurons expressing neuropeptide genes. $\boldsymbol{A}$, In situ hybridization revealed spatially restricted expression of achatin-I mRNA in the ventroposterior part of the pedal ganglia (arrowhead). B, Hybridization by sense probe. C, RT-PCR of achatin-I. SEG, Subesophageal ganglia. 18S rRNA served as an internal control. D, Restricted expression of the LYC prepropeptide gene in the right parietal ganglion revealed by in situ hybridization. $\boldsymbol{E}$, Hybridization by sense probe. $\boldsymbol{F}, \mathrm{A}$ BrdU-positive nucleus (green) of a neuron expressing achatin-I mRNA (red) observed with a fluorescence microscope. $\boldsymbol{G}$, Nuclear staining by DAPI (blue). $\boldsymbol{H}, \boldsymbol{I}$, Magnified images of $\boldsymbol{F}$ and $\boldsymbol{G}$ obtained with a confocal microscope. J, A BrdU-positive nucleus (green) of a neuron expressing LYC prepropeptide mRNA (red) observed with a fluorescence microscope. $\boldsymbol{K}$, Nuclear staining by DAPI (blue). $\boldsymbol{L}, \boldsymbol{M}$, A magnified image of $\boldsymbol{J}$ and $\boldsymbol{K}$ obtained with a confocal microscope. D, Dorsal; $V$, ventral; $L$ and Lat, lateral; $M$ and Med, medial; $C G$, cerebral ganglion. Scale bars: A, $250 \mu \mathrm{m} ; \mathbf{G}, \boldsymbol{K}, 125 \mu \mathrm{m}$. 
slugs. Based on in situ hybridization (Fig. $5 A, B$ ) and RT-PCR analyses (Fig. 5C) as well as a previous report on Achatina fulica (Satake et al., 1999), almost all the achatin-I mRNAs can be considered to be derived from the clusters located in the ventroposterior part of the pedal ganglia. We thus divided the number of achatin-I cDNAs per brain by the average number of achatin-I-positive neurons (192 and 204, respectively) (Fig. 6B) to calculate the number of achantin-I cDNAs per single neuron (Fig. 7A). The group data clearly showed that a larger number of achatin-I transcripts were contained in a single neuron in the growth-promoted slugs than in the growth-suppressed slugs (Fig. 7B).

\section{Discussion}

We have demonstrated that the sizes of the brain and neurons and the occurrence of DNA synthesis in the neurons all increase during body growth under a regulated dietary condition. The increase in the brain size was not accompanied by the increase in the number of neurons, supporting the view that the body growth results in DNA amplification within the nucleus of neurons. The increase in the brain/neuron sizes also brought about the increase in the amount of transcripts within a single neuron. This is the first quantitative demonstration that the body growth is accompanied by increases in DNA endoreplication and neuronal size in adult mollusk.

\section{Increase in the size of ganglia and neurons}

The volume measurement of each ganglion revealed that the increase was more prominent in the ganglia belonging to the subesophageal ganglia than in the PC (Fig. 2). Because the PC contains a large number of olfactory interneurons (Gelperin and Tank, 1990; Chase, 2000), our observation argues for the hypothesis by Gillette (1991) that the size of the body must be more faithfully reflected in the sizes of effector neurons than in the interneurons. In fact, we observed that the size of the neurons regulating the heart beat (LdWFamide-containing VGCs and achatin-I-containing neurons) was greater in the growthpromoted slugs than in the growth-suppressed slugs (Figs. $3 \mathrm{H}$, $6 A, B)$.

The MCGs are known to function as a regulator of the pattern generation of the feeding rhythm by innervating the buccal ganglion (Berry and Pentreath, 1976). The MCG is a modulatory interneuron in this sense, and our observation that MCG size correlates with the body weight (Fig. 3I) seems to be contradictory to the above notion. However, the MCG also functions as an effector motor neuron by synapsing onto the buccal musculature and other targets (Weiss and Kupfermann, 1976; Weiss et al., 1978). In fact, Berry and Pentreath (1976) have noticed that the MCGs also send extensive innervation to the periphery in Planorbis. Our observation indicates that the sizes of such multifunctional neurons are also under the influence of the body size.
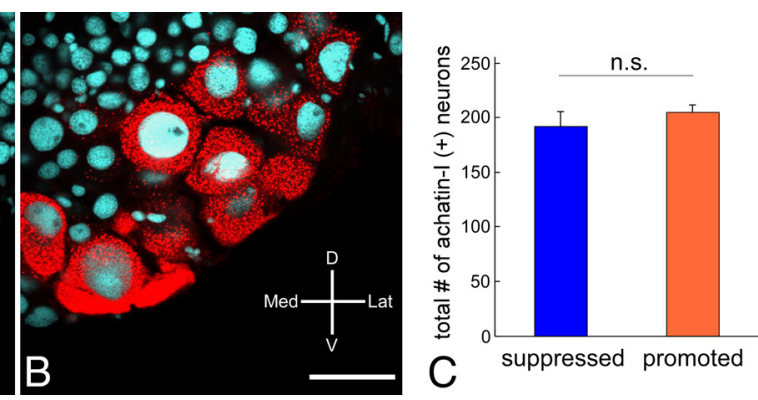

Figure 6. The number of achatin-I-expressing neurons was almost identical in the brains of the growth-suppressed and the growth-promoted slugs. $\boldsymbol{A}, \boldsymbol{B}$, Confocal microscopic images of coronal sections of the pedal ganglia of a growth-suppressed $(\boldsymbol{A})$ and a growth-promoted $(\boldsymbol{B})$ slug. These two images were obtained using the same magnification. Red, Positive signal of in situ $\mu \mathrm{m} . \boldsymbol{C}$, The mean total numbers of achatin-I-positive neurons in the growth-suppressed $(n=5)$ and growth-promoted $(n=5)$

A

Figure 7. The absolute copy numbers of achatin-I cDNA per single neuron. $\boldsymbol{A}$, The copy numbers of achatin-I cDNA in each brain determined by quantitative RT-PCR were divided by the average number of achatin-I-containing neurons (192 and 204) (Fig. 6B). $\boldsymbol{B}$, The group data of $\boldsymbol{A}$, showing a statistically significant difference between the growth-suppressed $(n=9)$ and growthpromoted $(n=7)$ slugs $\left({ }^{* *} p<0.01\right)$. Error bars indicate \pm SE.

\section{Enhanced expression of the achatin-I gene by growth promotion}

The amount of achatin-I transcripts was enhanced per neuron by growth promotion (Fig. 7). This seems to be caused, at least partly, by the amplification of the DNA region encompassing the achatin-I gene locus or endopolyploidy in the nuclei of achatinI-expressing neurons. There is, however, no denying the possibility that the transcriptional activity is also enhanced in the achatin-I gene. The combination of DNA endoreplication and transcriptional activation will result in the production of much larger amount of achatin-I protein products. Although we have examined only achatin-I-containing neurons in the present study, similar phenomena are expected in other motor and/or endocrine neurons in the brain.

It is intriguing to see whether the whole genome is repeatedly replicated (polyploidy) or only the necessary parts of the genome are amplified (polyteny) in nucleus. Single-cell quantitative genomic PCR will be one candidate method to uncover this issue. Our preliminary data showed that the several different genomic loci are amplified to the same extent in the VGCs compared with the sperm (haploid), supporting the notion that the VGCs are polyploid.

\section{DNA synthesis during body growth}

Since the discovery of giant neurons in the brains of gastropods, most researchers have analyzed the extent of DNA endoreplication by cytophotometric methods (Kuhlmann, 1969; Coggeshall et al., 1970; Boer et al., 1977). These analyses, however, do not 
reveal anything about when the DNA synthesis has occurred during the course of development and growth. We demonstrated by using the BrdU-labeling method that the DNA synthesis occurs even in adults at $\sim 14 \mathrm{~d}$ after the beginning of a dietary regulation period. Taking into account the fact that the average lifespan of slugs is $\sim 1$ year or more (Kano et al., 2001), our results indicate that DNA synthesis is triggered very quickly by body growth or improvement of nutrition.

Compared with the difference in the whole-brain size (the growth-suppressed vs the growth-promoted, 2.18-fold) (Fig. 2D, right), the difference in the DNA content in the whole brain was more modest (1.78-fold) (Fig. 4A). Although we still do not know the precise quantitative relationship between the size of the neuron and its DNA content, such apparent inconsistency can be explained by the fact that approximately one-half of the neurons of the brain are composed of PC neurons that contain a diploid genome within their nuclei (Chase and Tolloczko, 1987; Gelperin and Tank, 1990; Chase, 2000). This preponderance of diploid PC neurons would have reduced the average DNA content in the brain as a whole.

The scattered spatial distribution of BrdU-positive signals indicates that BrdU incorporation is not the result of cell division but of endoreplication. Our rough estimation of the total number of neurons in the left pleural ganglia also supports the idea that the ganglion volume is increased without increasing the number of neurons. However, there still remains a possibility that neurogenesis is also enhanced in the growth-promoted slugs in the other ganglia or at an undetectable level. This issue should to be more thoroughly investigated in future studies. We observed a larger difference in the number of BrdU-positive neurons between the growth-suppressed and the growth-promoted slugs in the subesophageal ganglia compared with the cerebral ganglion (Fig. 4D). This result is consistent with our observation that the size differences of ganglia were more prominent in the subesophageal ganglia than in the cerebral ganglia (Fig. 2D).

Among the multiple BrdU-positive neurons, we found several such neurons that express neuropeptide-encoding mRNAs whose protein products function as cardioexcitatory molecules (Fig. 5). It is not clear whether such peptidergic neurons are overrepresented among all BrdU-positive neurons, i.e., whether endoreplication occurs selectively in neurons that must keep their own sizes up to the body growth. However, taking into account that there are great differences in neuronal size among the neurons in the subesophageal ganglia (Gillette, 1991; Mandrioli et al., 2010) and that the enlargement of ganglia was more prominent in the subesophageal ganglia (Figs. $1 C, 2 D$ ), it is reasonable to suppose that there are some biases in the frequency of DNA endoreplication among the neuronal population during body growth.

\section{Endoreplication in large neurons}

Neurons are generally diploid in mammals, although several types of cells such as megakaryocytes or trophoblasts are known to be polyploid (Edgar and Orr-Weaver, 2001). As an exception, however, some of Purkinje cells in the cerebellum are proposed to be tetraploid, but this is still controversial (Lapham, 1968; Del Monte, 2006). Because Purkinje cell is one of the largest types of neurons in the mammalian brain, the tetraploidy of this neuron is well in accordance with the result of the present study. It will be intriguing to investigate histochemically using BrdU whether DNA endoreplication actually occurs in Purkinje cells in parallel with the body growth of mammals.

Finally, future efforts should be devoted to elucidating how the body growth or nutrition status was detected by motor neu- rons, resulting in the endoreplication of the nuclei. One possibility is that a larger amount of some neurotrophic factor(s) is retrogradely incorporated into motor neurons as the target tissue grows, and this triggers a molecular cascade, resulting in the DNA synthesis in these neurons.

\section{References}

Anisimov AP (2005) Endopolyploidy as a morphogenetic factor of development. Cell Biol Int 29:993-1004.

Berry MS, Pentreath VW (1976) Properties of a symmetric pair of serotonincontaining neurons in the cerebral ganglia of Planorbis. J Exp Biol 65: 361-380.

Boer HH, Montagne-Wajer C (1994) Functional morphology of the neuropeptidergic light-yellow-cell system in pulmonate snails. Cell Tissue Res 277:531-538.

Boer HH, Groot C, Dejong-Brink M, Cornelisse CJ (1977) Polyploidy in the freshwater snail Lymnaea stagnalis (Gastropoda, Pulmonata). A cytophotometric analysis of the NDA in neurons and some other cell types. Neth J Zool 27:245-252.

Chase R (2000) Structure and function in the cerebral ganglion. Microsc Res Tech 49:511-520.

Chase R, Tolloczko B (1987) Evidence for differential DNA endoreplication during the development of a moluscan brain. J Neurobiol 18:395-406.

Chomczynski P, Sacchi N (1987) Single-step method of RNA isolation by acid guanidinium thiocyanate-phenol-chloroform extraction. Anal Biochem 162:156-159.

Coggeshall RE, Yaksta BA, Swartz FJ (1970) A cytophotometric analysis of the DNA in the nucleus of the giant cell, R-2, in Aplysia. Chromosoma 32:205-212.

Cuoghi B, Mola L (2007) Morphological, cytochemical, and cytofluorimetric features of supramedullary neurons of the fish Solea ocellata. Biol Bull 212:1-5.

Del Monte U (2006) The puzzle of ploidy of Purkinje neurons. Cerebellum 5:23-26.

Edgar BA, Orr-Weaver TL (2001) Endoreplication cell cycles: more or less. Cell 105:297-306.

Fukunaga S, Matsuo R, Hoshino S, Kirino Y (2006) Novel kruppel-like factor is induced by neuronal activity and by sensory input in the central nervous system of the terrestrial slug Limax valentianus. J Neurobiol $66: 169-181$.

Gelperin A, Tank DW (1990) Odour-modulated collective network oscillations of olfactory interneurons in a terrestrial mollusc. Nature 345:437-440

Gillette R (1991) On the significance of neuronal giantism in gastropods. Biol Bull 180:234-240.

Kano Y, Fukuda H, Yoshizaki H, Saito M, Hosaka K, Sugimura T, Ito YF, Fujiwara H, Nakamura Y, Mashino K, Ito K, Tone K, Fukuda T, Mitoki T, Yamashita H, Hori S, Hori K, Hori H (2001) Distribution and seasonal maturation of the alien slug Lehmannia valentiana (Gastropoda: Pulmonata: Limacida) in Yamaguchi Prefecture, Japan. The Yuriyagai 8:1-13.

Kuhlmann D (1969) Determination of the DNA content in the cell nuclei of the nerve tissue of Helix pomatia L. and Panorbarius corneus L. (Atylomatophora and Basommatophora, Gastropoda). Experimentia 25:848-849.

Lapham LW (1968) Tetraploid DNA content of Purkinje neurons of human cerebellar cortex. Science 159:310-312.

Lasek RJ, Dower WJ (1971) Aplysia californica: analysis of nuclear DNA in individual nuclei of giant neurons. Science 172:278-280.

Mandrioli M, Mola L, Cuoghi B, Sonetti D (2010) Endoreplication: a molecular trick during animal neuron evolution. Q Rev Biol 85:159-169.

Matsuo R, Ito E (2011) Spontaneous regeneration of the central nervous system in gastropods. Biol Bull, in press.

Matsuo R, Asada A, Fujitani K, Inokuchi K (2001) LIRF, a gene induced during hippocampal long-term potentiation as an immediate-early gene, encodes a novel RING finger protein. Biochem Biophys Res Commun 289:479-484.

Matsuo R, Kobayashi S, Watanabe S, Namiki S, Iinuma S, Sakamoto H, Hirose K, Ito E (2009) Glutamatergic neurotransmission in the procerebrum (olfactory center) of a terrestrial mollusk. J Neurosci Res 87:3011-3023.

Matsuo R, Kobayashi S, Murakami J, Ito E (2010) Spontaneous recovery of 
the injured higher olfactory center in the terrestrial slug Limax. PLoS One 5:e9054.

Mola L, Cuoghi B, Mandrioli M, Marini M (2001) DNA endoreplication in the clustered supramedullary neurons of the pufferfish Diodon holacanthus L. (Osteichthyes). Histochem J 33:59-63.

Morishita F, Nakanishi Y, Kaku S, Furukawa Y, Ohta S, Hirata T, Ohtani M, Fujisawa Y, Muneoka Y, Matsushima O (1997) A novel D-amino-acidcontaining peptide isolated from Aplysia heart. Biochem Biophys Res Commun 240:354-358.

Morishita F, Sasaki K, Kanemaru K, Nakanishi Y, Matsushima O, Furukawa Y (2001) NdWFamide: a novel excitatory peptide involved in cardiovascular regulation of Aplysia. Peptides 22:183-189.

Morishita F, Nakanishi Y, Sasaki K, Kanemaru K, Furukawa Y, Matsushima O (2003a) Distribution of the Aplysia cardioexcitatory peptide, NdWFamide, in the central and peripheral nervous system of Aplysia. Cell Tissue Res 312:95-111.

Morishita F, Minakata H, Sasaki K, Tada K, Furukawa Y, Matsushima O, Mukai ST, Saleuddin AS (2003b) Distribution and function of an Aplysia cardioexcitatory peptide, NdWFamide, in pulmonate snails. Peptides 24:1533-1544.

Sambrook J, Russell DW (2001) Molecular cloning: a laboratory manual, Ed 3. Cold Spring Harbor, NY: Cold Spring Harbor Laboratory.

Satake H, Yasuda-Kamatani Y, Takuwa K, Nomoto K, Minakata H, Nagahama T, Nakabayashi K, Matsushima O (1999) Characterization of a
cDNA encoding a precursor polypeptide of a D-amino acid-containing peptide, achatin-I and localized expression of the achatin-I and fulicin genes. Eur J Biochem 261:130-136.

Shirahata T, Watanabe S, Kirino Y (2004) Distribution of serotonin-like immunoreactive neurons in the slug Limax valentianus. Cell Tissue Res 315:285-290.

Udaka H, Numata H (2008) Short-day and low-temperature conditions promote reproductive maturation in the terrestrial slug, Lehmannia valentiana. Comp Biochem Physiol A Mol Integr Physiol 150:80-83.

Watanabe S, Kirino Y, Gelperin A (2008) Neural and molecular mechanisms of microcognition in Limax. Learn Mem 15:633-642.

Weiss KR, Kupfermann I (1976) Homology of the giant serotonergic neurons (metacerebral cells) in Aplysia and pulmonate molluscs. Brain Res 117:33-49.

Weiss KR, Cohen JL, Kupfermann I (1978) Modulatory control of buccal musculature by a serotonergic neuron (metacerebral cells) in Aplysia. J Neurophysiol 41:181-203.

Yeoman MS, Brierley MJ, Benjamin PR (1996) Central pattern generator interneurons are targets for the modulatory serotonergic cerebral giant cells in the feeding system of Lymnaea. J Neurophysiol 75:11-25.

Zakharov IS, Hayes NL, Ierusalimsky VN, Nowakowski RS, Balaban PM (1998) Postembryonic neurogenesis in the procerebrum of the terrestrial snail, Helix lucorum L. J Neurobiol 35:271-276. 